\title{
Gaseous Plasma's Time-behavior on a Plate Motion Damping with Time Influenced by a Non-Uniform Non-Stationary Electric Field.
}

Taha Zakaraia Abdel Wahid ( $\sim$ taha_zakaraia@yahoo.com )

Menoufia University https://orcid.org/0000-0003-3786-5393

\section{K. Hadouda}

, El Gazeera High Institute for Engineering and Technology, Cairo, Egypt.

\section{Research Article}

Keywords: Gaseous Plasma, Unsteady analytical solution, External electric field, Irreversible nonequilibrium thermodynamics, Boltzmann Kinetic equation, Maxwell equations, Entropy, Internal energy, Nonlinear electromagnetic field

Posted Date: January 28th, 2022

DOI: https://doi.org/10.21203/rs.3.rs-1304448/v1

License: (c) (i) This work is licensed under a Creative Commons Attribution 4.0 International License. Read Full License 


\title{
Time-behavior of Gaseous Plasma on a Plate Motion Damping with Time Influenced by a Non-Uniform Non-Stationary Electric Field.
}

\author{
T. Z. Abdel Wahid ${ }^{1}$, M. K. Hadouda ${ }^{2}$ \\ ${ }^{1}$ Mathematics and Computer Science Department, Faculty of Science, Menoufia University, Shebin El-Kom 32511, Egypt. \\ ${ }^{2}$ Basic Sciences Department, El Gazeera High Institute for Engineering and Technology, Cairo, Egypt. \\ Corresponding author: T. Z. Abdel Wahid (e-mail: taha_zakaraia@yahoo.com).
}

ABSTRACT The research subject is the kinetic and irreversible thermodynamic behavior of gaseous plasma (GP) flow limited by a moving rigid flat plate (RFP). The effects of an unsteady nonlinear applied electric field (NLAEF) were examined on the GP. To explore gas dynamics with the electron velocity distribution function (EVDF), researchers have concentrated on the Bhatnagar-Gross-Krook (BGK)-model of the kinetic Boltzmann equation (BE). An analytical solution was found using the moment method (MM), traveling wave, and shooting method. As illustrated, mean velocity, shear stress, and electromagnetic fields all founded play essential roles. An interesting comparison between the non-equilibrium EVDF and the equilibrium EVDF is made carefully with 3-Dimensional graphics in various time values. We found that the system goes to an equilibrium state (ES) with time compatible with Le Chatelier's principles. The relations between the various macroscopic variables of the GP are studied. The irreversible non-equilibrium thermodynamics (NT) properties of the system are presented. Entropy and entropy generation are derived, and their behavior is investigated. The essence of entropy, the degree of internal chaos of a system, is gradually described with the advent of statistical physics and information theory. Cybernetics, probability theory, life science, and astrophysics are just a few of the domains where it is functional. According to our findings, NLAEF has a strong effect on GP. Compared to the effect of the nonlinear applied magnetic field (NLAMF), it causes it to vary and disturb substantially. To save the ES for a GP, we should employ NLAMF rather than NLAEF in the GP management procedure. The importance of this research stems from its wide applications in domains such as physics, electrical engineering, micro-electro-mechanical systems (MEMS), and nano-electro-mechanical systems (NEMS) technologies as industrial and commercial sectors.

\section{PACS numbers: 52.30.-q, 71.10.Ca, 52.35.Sb, 52.25.Kn.}

INDEX TERMS Gaseous Plasma; Unsteady analytical solution; External electric field; Irreversible nonequilibrium thermodynamics; Boltzmann Kinetic equation; Maxwell equations; Entropy, Internal energy, Nonlinear electromagnetic field.

\section{INTRODUCTION}

The BE has several benefits in MEMS \& NEMS technology. One of the most necessary concerns driving the usage of the BE in MEMS \& NEMS performances is the understanding that: Since their micronscale size is ordinarily relative to the molecule mean free path underneath normal operating conditions. Thus, the Knudsen flow numbers in MEMS \& NEMS are commonly far from the continuum rules. Microflow is a term utilized to define flow on a micron scale. The usual lengths of the flow gradient in microflows are usually modest and approximate to the molecules' mean free path. Often these MEMS \& NEMS regular lengths will be in the micron ordering or smaller, resulting in a Knudsen number between 0.001 to 10 . Outcoming fluid flows in the slip and transition flow requirements like most MEMS \& NEMS technology. Microflows in these rules have features that counter typical flows with long-expected lengths. The Navier-Stokes and other standard continuum measures cannot characterize and predict micro and nano-flows. Moreover, microflows affect the efficacy of MEMS \& NEMS, like as micro pressure detectors, micro pumps, and valves [1]. 
Low-temperature GP or vacuum GP technologies have been used extensively in semiconducting and circuit boards for more than four decades. They are now widely employed in magnetic media, metallic treatment, and cleaning, such as removing carbon following laser machining. By reengineering the surface characteristics of polymeric materials, these systems achieve new industrial implementations in microelectronics and photonics, allowing these industries to modify the chemical and physical properties of the material surface without affecting the bulk properties of the entire material [1-4]. The temperature, $T_{a}$, of low-temperature plasmas, can be represented as $T_{i} \approx T_{n} \approx T_{e}$, where $T_{i}$ is the temperature of the ions, $T_{e}$ is the temperature of the electrons, and $T_{n}$ is the temperature of the neutral atom. These characteristics help create GP-based technology that employs various electron and ion MVDFs. They characterize the kinetic behavior of the GP by conveying the energy accessible to large species through multiple collisional phenomena (electron-electron, electron-ion, and electron-neutral). As a result, it is critical to compare the electrons' EVDF with the EC and modify it to follow the electron behavior. It is generally known that the $\mathrm{BE}$ is often used to characterize GP mobility in compressional GP when microscopic factors are considered. Many analytical and experimental contributions [6], [7] are concerned with creating approaches for obtaining realistic solutions to BE. The Bhatnagar-Gross-Krook approach for the BE, or the BGK-type [8-10], is well known as the most impressive approximation of the difficult collision factor in BE. As a result, various trials employing MM [11]-[12] have been established to handle the BE depending on the BGK-type.

Greater mean free path values sufficiently explained the rarefied gases for ionized flows throughout various Knudsen numbers $(K n \sim 0.1-10)$. Modified values of the recognized length in MEMS and NEMS technologies are widely utilized in various commercial activities (see Refs. [11-15]). In GP technology, particularly in low-temperature GP, the contact between flowing GP and rigid surfaces is critical. The ionization degree of charged particles is modest, with a single charged particle having between 100,000 and 1,000,000 neutrals. As a result, instability, discharge management, and the creation of excited atoms are all handled by ions.

For example, Ref. [15] investigates the flow dynamics of GP over RFPs using the BE. Furthermore, [16][29] have investigated GP flow as a rarefied electron gas and noble gases produced by neutral atoms. They used a numerical solution of the collision frequencies to characterize the behavior of the charged particle. For the Vlasov-Poisson-Landau system, Yan [30] presented a Hybrid methodology combining Monte Carlo, Particles in Cell, and Macro-Micro decomposed techniques. Pan et al. [31] examined the probability EVDF of the gas in discontinuous velocity space utilizing the finite volume approach, using charged particle transportation in the accelerating electric term of the BGK-type.

Juno et al. [32] used finite elements and Runge-Kutta algorithms to examine a novel method for 6D GP to discretize the Vlasov-Maxwell system to find new EVDF alternatives. Using a preservation kinetic gas method, Liu et al. [33] devised a new approach for the Vlasov-Poisson-BGK problem with large Kn and Debye length quantities. Now we are looking into the influence of electron-ion-neutral collisions in the BGK-kind and then calculating the exact solutions for the problem. Furthermore, we handle the entire BGK-kind collision frequency to prevent model discontinuities without any cut-off based on the total values of the independent variable [16]-[29]. The system of equations was solved with several assumptions in these studies.

The BE's advantage is that it allows us to examine the concepts of irreversible non-equilibrium thermodynamics (INTD). According to our findings, the displacement current associated with the NLAEF cannot be negated in the problem therapy. The non-stationary oscillating flow is studied by Abdel Wahid and Morad [34] in an INTD environment using the Stokes issue. Under the NLAEF effect, we wanted to employ the GP model next to a boundless oscillating RFP. The BGK-kind model was combined with the Maxwell model. Furthermore, two-sided MVDFs were used with MM and traveling wave solutions.

Finally, theoretical descriptions of electromagnetic fields, viscosity coefficient, velocity, and shear stress were provided. The difference in variance between the MVDFs and the perturbed MVDFs was shown. 
Because of the BE's characteristics, we investigated INTD concepts. The findings acquired were in good accord with those found in [34].

\section{GEOMETRY AND GOVERNING EQUATIONS OF THE PROBLEM}

We analyze a new helium GP system with a non-uniform applied electric field $E_{x A}$ parallel to the flow direction positioned near an infinite horizontally RFP $y=0$ bordered by the upper half-space at $y \geq$ 0 . Initially, the helium GP is contained in an EC. It has been hypothesized that the GP is isothermal $T_{e} \approx$ $T_{i}$ and quasi-neutral. We will refer to the NLAEF direction on the $\mathrm{x}$-axis and the magnetic field on the $\mathrm{z}$ axis. The GP flows to an unstable NLAEF, and the surface RFP moves with a velocity equal to $V_{0} e^{-\omega t}$ along the x-axis. Furthermore, the reflected particles acquired RFP velocity in every case.

The system is studied using the Lorentz force $\vec{F}_{e}$ that is acting on the system components as electrons and ions at a constant temperature

$\vec{F}_{e}=-e \vec{E}-\frac{e}{c}(\vec{\eta} \wedge \vec{B})$, here $\vec{E}=E_{x} \vec{\imath}=\left(E_{x A}+E_{x i}\right) \vec{l}$, as $E_{x A}=E_{0} e^{\phi_{0}\left(k_{1} y+k_{2} t+k_{0}\right)}$

The applied non-uniform unstable electric field and the induced one are represented by $E_{x A}$ and $E_{x i}$, respectively, and the $k_{0}, k_{1}$, and $k_{2}$ constants have restricted values to prevent the electric field from diverging.

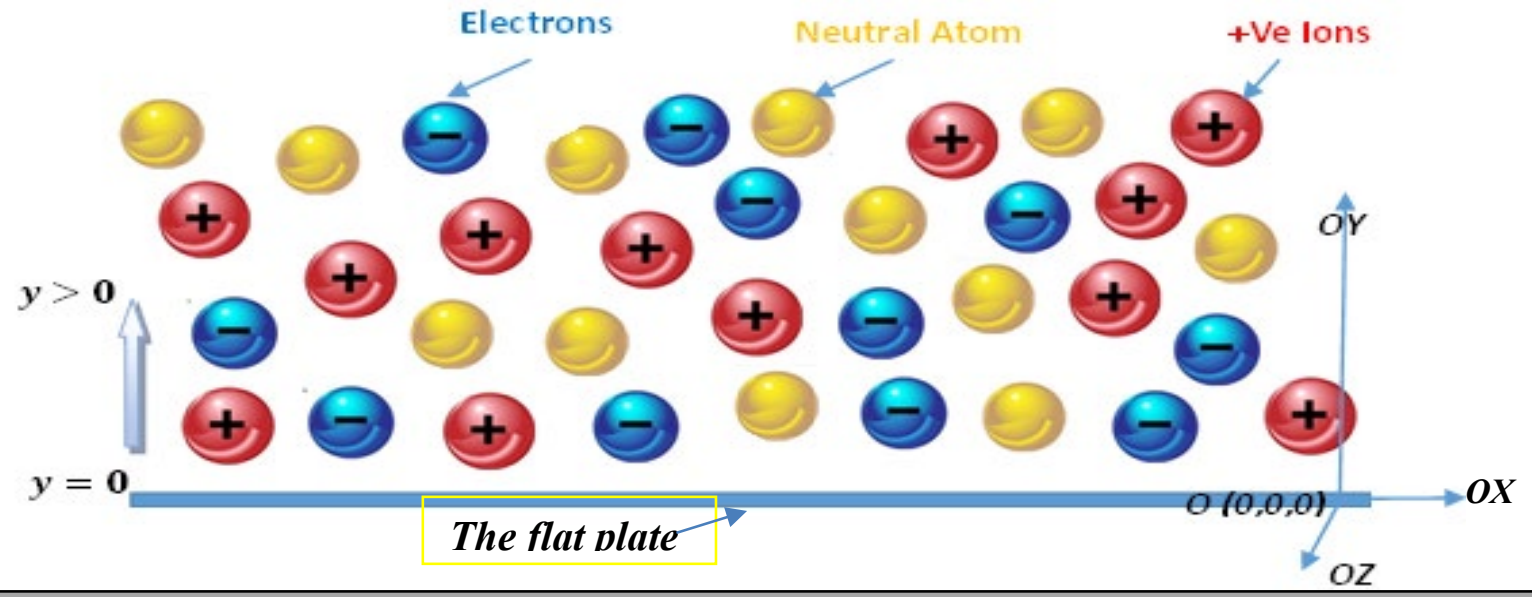

Figure 1. Schematic representation of our problem. The rigid flat plate located at $y=0$ and the plasma components occupied the half-space $y>0$.

The Maxwell equations are satisfied by the velocity, flux, electric, and magnetic fields as functions of $y$ and $t$ and can be represented as

$\vec{V} \equiv\left(V_{x}, 0,0\right), \vec{J} \equiv\left(q n V_{x}, 0,0\right), \vec{E} \equiv\left(E_{x}, 0,0\right)$ and $\vec{B} \equiv\left(0,0, B_{z}\right)$

Using the kinetic characterization of GP, the electrons EVDF $g_{e}(\vec{r}, \vec{\eta}, t)$ linked to helium-GP can be examined theoretically. We analyze the well-known BE-like kinetic equation with a collision term of the BGK-type, which models the EVDF in collisions [35], [36]:

$\frac{\partial g_{e}(\vec{r}, \vec{\eta}, t)}{\partial t}+\vec{\eta} \cdot \frac{\partial g_{e}}{\partial \vec{r}}+\frac{\vec{F}_{e}}{m_{e}} \cdot \frac{\partial g_{e}}{\partial \vec{\eta}}=\omega_{e e}\left(g_{0 e}-g_{e}\right)+\omega_{e i}\left(g_{0 i}-g_{e}\right)+\omega_{e n}\left(g_{0 n}-\right.$

$\left.g_{e}\right)$ as $g_{0 \alpha}=n_{\alpha}\left(2 \pi R T_{\alpha}\right)^{-\frac{3}{2}} e^{\frac{-\left(\vec{\eta}-\vec{V}_{\alpha}\right)^{2}}{2 R T_{\alpha}}}$ 
The variables $n_{\alpha}, \vec{V}_{\alpha}$, and $T_{\alpha}$, distinguish the significant characteristics of the model can be obtained using MM. The beginning and boundary conditions can be represented as $[16,17,29,46]$ based on the geometry of the issue:

$V_{x 2}(0, t)=V_{0} e^{-\omega t}$ at $t>0, V_{x}$ is finite at $y \rightarrow \infty$, and $V_{x}=V_{x 2}$ at $\eta_{y}>0$.

These hypotheses will translate the GP kinetic equation into a proper shape.

$\frac{\partial g_{e}}{\partial t}+\eta_{y} \frac{\partial g_{e}}{\partial y}-\frac{e B_{e_{z}}}{m_{e} c}\left(\eta_{y} \frac{\partial g_{e}}{\partial \eta_{x}}-\eta_{x} \frac{\partial g_{e}}{\partial \eta_{y}}\right)+\frac{e E_{e_{x}}}{m_{e}} \frac{\partial g_{e}}{\partial \eta_{x}}=\omega_{e e}\left(g_{0 e}-g_{e}\right)+\omega_{e i}\left(g_{0 i}-g_{e}\right)+\omega_{e n}\left(g_{0 n}-g_{e}\right)$

$\omega_{e e}, \omega_{e i}$, and $\omega_{e n}$ collision frequencies take the form [37], [38]:

$\omega_{e e}=\left(\frac{4 \sqrt{\pi} n_{e} e^{4} \log \left[\Lambda_{e e}\right]}{3 \sqrt{m_{e}} K_{B}{ }^{\frac{3}{2}} T_{e}^{\frac{3}{2}}}\right), \omega_{e i}=\left(\frac{4 \sqrt{2 \pi} n_{i} e^{4} Z^{2} \log \left[\Lambda_{e i}\right]}{3 \sqrt{m_{e}} K_{B}{ }^{\frac{3}{2}} T_{e}^{\frac{3}{2}}}\right), \omega_{e n}=\left(\frac{4 \sqrt{\pi m_{n}} n_{e} e^{4} \log \left[\Lambda_{e n}\right]}{3 m_{e} K_{B} K^{\frac{3}{2}} T_{e}^{\frac{3}{2}}}\right)$

Here, $\lambda_{D e}=\lambda_{D i}=\lambda_{D}, \log [\Lambda]=\log \left[4 \pi n \lambda^{3}{ }_{D}\right]$, and $Z$ are the Debye lengths, the Coulomb logarithm, and the ionization degree.

Lee's approach for inherently unknowable functions of time and space, $V_{x 1}$ and $V_{x 2}$ [38], [39], can be used to solve Eq. (4):

$g=\left\{\begin{array}{l}g_{1}=n(2 \pi R T)^{-\frac{3}{2}}\left(1+\frac{\eta_{x} V_{x 1}}{R T}\right) e^{\left(\frac{-\eta^{2}}{2 R T}\right)} \text { for } \eta_{y}<0 \downarrow, \\ g_{2}=n(2 \pi R T)^{-\frac{3}{2}}\left(1+\frac{\eta_{x} V_{x 2}}{R T}\right) e^{\left(\frac{-\eta^{2}}{2 R T}\right)} \text { for } \eta_{y}>0 \uparrow\end{array}\right.$

Using the Grad MM with Eq. (4), we obtain the transfer equations for electrons [6]

$$
\frac{\partial}{\partial t} \int \vartheta_{j} g_{e} d \underline{\eta}+\frac{\partial}{\partial y} \int \eta_{y} \vartheta_{j} g_{e} d \underline{\eta}+\frac{e E_{x e}}{m_{e}} \int g_{e} \frac{\partial \vartheta_{j}}{\partial \eta_{x}} d \underline{\eta}-\frac{e B_{z e}}{m_{e} c} \int\left(\eta_{x} \frac{\partial \vartheta_{j}}{\partial \eta_{y}}-\eta_{y} \frac{\partial \vartheta_{j}}{\partial \eta_{x}}\right) d \underline{\eta}=
$$

$\omega_{e e} \int \vartheta_{j}\left(g_{0 e}-g_{e}\right) d \underline{\eta}+\omega_{e i} \int \vartheta_{j}\left(g_{0 i}-g_{e}\right) d \underline{\eta}+\omega_{e n} \int \vartheta_{j}\left(g_{0 n}-g_{e}\right) d \underline{\eta}$

The equivalent relation of the integrals of the functions $\vartheta_{j}(\vec{\eta})$ overd $\underline{\eta}=d \eta_{x} d \eta_{y} d \eta_{z}$,

$\int \vartheta_{j}(\vec{\eta}) g d \underline{\eta}=\int_{-\infty}^{\infty} \int_{-\infty}^{0} \int_{-\infty}^{\infty} \vartheta_{j} g_{1} d \underline{\eta}+\int_{-\infty}^{\infty} \int_{0}^{\infty} \int_{-\infty}^{\infty} \vartheta_{j} g_{2} d \underline{\eta}$

The Maxwell equations can be expressed for electrons as follows:

$$
\begin{aligned}
& \frac{\partial E_{x e}}{\partial y}-\frac{1}{c} \frac{\partial B_{z e}}{\partial t}=0, \\
& \frac{\partial B_{z e}}{\partial y}-\frac{1}{c} \frac{\partial E_{x e}}{\partial t}-\frac{4 \pi e n_{e}}{c} V_{x e}=0 .
\end{aligned}
$$

Observe that the current displacement component in the Maxwell equations is factored into the equation, although in prior research, it was omitted; see [17], [18], [20], [26], [28].

The electromagnetic fields can be computed using the standard conditions.

$E_{x}(y, 0)=E_{0}, B_{z}(y, 0)=0, E_{x}$ and $B_{z}$ are finite at $\mathrm{y} \rightarrow \infty$.

The non-dimensional variables can now be defined as follows:

$V_{T h}=\sqrt{2 R T}, \omega=\frac{\omega^{*}}{\tau_{e e}}$.

$$
\begin{gathered}
t=t^{*} \tau_{e e}, y=y^{*}\left(\tau_{e e} V_{T h}\right), V_{x}=V_{x}^{*} V_{T h}, \tau_{x y}=\tau_{x y}^{*} V_{T h}, M=\frac{V_{0}}{V_{T h}}, E_{x}=E_{x}^{*}\left(\frac{m_{e} c}{e \tau_{e e}}\right), \\
B_{z}=B_{z}^{*}\left(\frac{m_{e} c}{e \tau_{e e}}\right), \gamma=\frac{m_{e}}{m_{i}}, d U=d U^{*}\left(K_{B} T_{e}\right) \text { and } g_{l}=g_{l}^{*} n_{e}\left(2 \pi R T_{e}\right)^{-\frac{3}{2}}, l=0,1,2,
\end{gathered}
$$

The mean velocity and shear stress take the form [38]:

$V_{x}=\frac{1}{2}\left(V_{x 1}+V_{x 2}\right), \tau_{x y}=\frac{P_{x y}}{\rho V_{0} \sqrt{R T_{e} / 2 \pi}}=\left(V_{x 2}-V_{x 1}\right) \cdot \operatorname{Here} P_{x y}=m \int\left(\eta_{x}-V_{x}\right) \eta_{y} g d \underline{\eta}$

The Mach number $(M)$ value is 0.01 , then the modification $n$ and $T$ is minimal and $n_{\alpha}=1+O\left(M^{2}\right)$ $T_{\alpha}=1+O\left(M^{2}\right)$. Applying the non-dimensional values and this hypothesis (12) along with $\vartheta_{1}=\eta_{x}$ and $\vartheta_{2}=\eta_{x} \eta_{y}$ the transfer equations (7) for electrons will be 
$\frac{\partial V_{e x}^{*}}{\partial t^{*}}+\frac{\partial \tau_{e x y}^{*}}{\partial y^{*}}-E_{e x}^{*}=0$

$\frac{\partial \tau_{e x y}^{*}}{\partial t^{*}}+2 \pi \frac{\partial V_{e x}^{*}}{\partial y^{*}}+\tau_{\text {exy }}^{*}=0$

In this scenario, the initials and boundary conditions will be:

$\left.\begin{array}{c}V_{e x}^{*}\left(y^{*}, 0\right)=\tau_{\text {exy }}^{*}\left(y^{*}, 0\right)=0, \\ 2 V_{e x}^{*}\left(0, t^{*}\right)+\tau_{\text {ex }}^{*}\left(0, t^{*}\right)=2 M e^{-\omega^{*} t^{*}} \\ V_{e x}^{*} \text { and } \tau_{\text {exy }}^{*} \text { are finite as } \mathrm{y} \rightarrow \infty\end{array}\right\}$

\section{METHOD OF SOLUTION FOR THE NONDIMENSIONALIZED SYSTEM}

The boundary value issue for electrons will be simplified by removing the star from the non-dimensional parameters.

$\frac{\partial V_{e x}}{\partial t}+\frac{\partial \tau_{e x y}}{\partial y}-E_{e x}=0$

$\frac{\partial \tau_{e x y}}{\partial t}+2 \pi \frac{\partial V_{e x}}{\partial y}+\left(1+\frac{\omega_{e i}}{\omega_{e e}}+\frac{\omega_{e n}}{\omega_{e e}}\right) \tau_{e x y}=0$,

$\frac{\partial E_{e x}}{\partial y}-\frac{\partial B_{e z}}{\partial t}=0$

$\frac{\partial B_{e z}}{\partial y}-\frac{\partial E_{e x}}{\partial t}-\Omega_{e 0} V_{e x}=0$.

where $\Omega_{e 0}=\left(\frac{V_{T h}^{2} n_{e} e^{2}}{m_{e} C^{2} \omega_{e e}^{2}}\right)$.

By solving the BE using the traveling wave approach, the electron kinetics can be explained in total, and the new item can be represented as follows: [40] - [42]:

$\gamma=k_{1} y+k_{2} t+k_{0}$

Here $k_{1}, k_{2}$ and $k_{0}$ are the transformation constants.

The partial derivatives of Eqs. (17)- (20) can be determined from Eq. (21) as follows:

$\frac{\partial}{\partial t}=k_{2} \frac{\partial}{\partial \gamma}, \frac{\partial}{\partial y}=k_{1} \frac{\partial}{\partial \gamma}, \frac{\partial^{n}}{\partial t^{n}}=k_{2}^{n} \frac{\partial^{n}}{\partial \gamma^{n}}, \frac{\partial^{n}}{\partial y^{n}}=k_{1}^{n} \frac{\partial^{n}}{\partial \gamma^{n}}$.

Here $n$ is a positive integral number.

Substituting Eqs. (21)-(22) inside Eqs. (17)-(20), we have the following differential equation

$\left(\left(k_{2}^{2}-k_{1}^{2}\right)\left(k_{2}-\frac{2 \pi k_{1}^{2}}{k_{2}}\right)\right) \frac{d^{3} V_{e x}(\gamma)}{d \gamma^{3}}+\tau_{c}\left(k_{2}^{2}-k_{1}^{2}\right) \frac{d^{2} V_{e x}(\gamma)}{d \gamma^{2}}-\Omega_{e 0} k_{2} \frac{d V_{e x}(\gamma)}{d \gamma}+\Omega_{e 0} \tau_{c} V_{e x}(\gamma)=0, \tau_{c}=$

$\left(1+\frac{\omega_{e i}}{\omega_{e e}}+\frac{\omega_{e n}}{\omega_{e e}}\right)$.

In its dimensionless form, this equation can be solved with the following initial and boundary conditions:

$\left\{\begin{array}{c}B_{e z}(\gamma=0)=\tau_{e x y}(\gamma=0)=0, E_{e x}(\gamma=0)=E_{0} ; \\ 2 V_{e x}\left(\gamma=k_{2}\right)+\tau_{e x y}\left(\gamma=k_{2}\right)=2 M e^{-\omega} \text { at } y=0 \text { and } t=1 ; \\ V_{e x}, \tau_{e x y}, E_{e x}, \text { and } B_{e z} \text { are finite at } \gamma \rightarrow-\infty .\end{array}\right.$

In addition, given equation (1), it is noted that, for finite $y>0$ and finite time intervals, $t$ of practical interest, the applied electric field, $E_{x E}$, remains finite.

We can now solve the governing equations defining the research lab helium GP using symbolic software (Mathematica) with the initial and boundary conditions.

\section{THERMODYNAMIC TREATMENT OF HELIUM-PLASMA MODEL}

This section examines the non-equilibrium thermodynamic features of the helium GP while considering the problem's irreversible phenomena. The thermodynamic characteristics of the GP components will be determined by using EVDF in Eq. (6) and the collision frequencies of the GP 
constituents (ions, electrons, and neutrals). In addition, we will see if our approach is coherent with several thermodynamic principles and the H-theorem for the helium GP. In physics, entropy is derived from thermodynamics. It is one of the state parameters of matter used to characterize how energy is degraded. It refers to a measurement of the status of some material systems in general. The essence of entropy, the degree of internal chaos of a system, is gradually described with the advent of statistical physics and information theory. Cybernetics, probability theory, life science, and astrophysics are just a few of the domains where it is functional.

\section{A. ENTROPY AND ITS RELATED VARIABLES.}

According to [16]-[18], [43]: The entropy per unit mass $S$ and the y-component of entropy flux have the general structure:

$S=-\int g_{e} \ln g_{e} d \underline{\eta}=-\left(\int g_{e 1} \ln g_{e 1} d \underline{\eta}+\int g_{e 2} \ln g_{e 2} d \underline{\eta}\right)=-\pi^{\frac{3}{2}}\left[\left(V_{x 1}{ }^{2}+V_{x 2}{ }^{2}\right)-0.66\right]$,

$J_{y}{ }^{(S)}=-\int \eta_{y} g_{e} \ln g_{e} d \underline{\eta}=-\left(\int \eta_{y} g_{e 1} \ln g_{e 1} d \underline{\eta}+\int \eta_{y} g_{e 2} \ln g_{e 2} d \underline{\eta}\right)=\left[\pi\left(V_{x 1}{ }^{2}+V_{x 2}{ }^{2}\right)\right]$.

The entropy production can be calculated when using these principles:

$\sigma=\frac{\partial S}{\partial t}+\vec{\nabla} \cdot \overrightarrow{J(S)}$

It is needed by Boltzmann's H-theorem that entropy is an increasing function concerning time and that entropy production is often a nonnegative quantity [16-18, 43].

\section{B. GIBBS FORMULA AND ITS CONSEQUENCES}

The Gibbs formula can calculate the overall system's internal energy modification (IEM) [44]. The two significant types of helium GP magnetization are paramagnetic and diamagnetic. As a result, for both kinds, the total energy alterations can be stated as follows:

IEM in Gibbs' equation is described by the extensive factors $S, P, B$ related to intensive factors $T, E, M_{B}$, accordingly. As a result, the Gibbs law's IEM can be expressed as [29], [44]:

$d U=d U_{S}+d U_{p o l}+d U_{d i a}$, such that the IEM as a result of the modification in the entropy is $d U_{S}=$ $T d S$, the IEM as a result of the modification in polarization is $d U_{p o l}=E d P$, the IEM as a result of the modification in the magnetic field is $d U_{d i a}=-M_{B} d B$, with $M_{B}=T \frac{\partial S}{\partial B}$. The IEM in Gibbs' formulation was represented with the extended thermodynamic characteristics $S, P$, and $M$ because of $T$, $E$, and $B$, correspondingly. As a result, the Gibbs law's IEM can be expressed as:

$d U=d U_{S}+d U_{\text {pol }}+d U_{\text {par }}$. The IEM as a result of the modification in magnetization is $d U p a r a=$ $B d M$.

Finally, we can write the total energy modification as $d U=d S_{e}+f_{1} E_{x} d p+f_{1} B_{z} d M_{B}$. In this case, the IEM in a non-dimensional form (after dropping the star) will be in the modified Gibbs compact formula $d U=d S+E d p-M_{B} d B$.

\section{CALCULATIONS AND RESULTS OF THE PLASMA COMPOSITION}

This part provides an example of FINDINGS in GP [45]. By solving the BGK-kind for helium GP predicated on the INTD utilizing the traveling wave methodology for the $\omega_{e e}, \omega_{e i}$, and $\omega_{e n}$ colliding frequencies, the GP kinetics can be characterized in depth. The findings are displayed in figures and graphs utilizing a wide variety of standard data for electron gas in helium GP. In the experimental context, the findings in the figures reflect the normal verification consistency between BE and research lab GP estimates [20], [37]. The helium GP loses single electrons in the paramagnetic condition. However, the helium-GP loses electron pairs due to the atoms' ionizing potential in the diamagnetic scenario. We investigate the accurate solution of the system of equations (17-20) and Eq. (22) using the numerical 
shooting technique for $k_{1}=0.83, k_{2}=0.15, k_{0}=0.1$ and the surface Mach number, $M=10^{-2}$. The problem's computations are performed using various GP parameters and constraints:

The electron rest mass and the charge of the electron are $m_{e}=9.093 \times 10^{-28} \mathrm{gm}, e=4.810^{-10} \mathrm{esu}$. Boltzmann's constant, the initial temperature, and the electrons concentration are $k_{B}=1.3807 \times 10^{-16} \mathrm{erg} / \mathrm{K}, T_{0}=300 \mathrm{~K}, n_{e}=10^{11} \mathrm{~cm}^{-3}$. The atom diameter of the helium-GP is $d=$ $1.4 \times 10^{-8} \mathrm{~cm}$, and the dimensionless control parameter $\Omega_{e 0}=2.9 \times 10^{-6}$. The mean free path is $\lambda=$ $11.483 \times 10^{3} \mathrm{~cm}$, which is large compared with the Debye length $\lambda_{D e}=3.779 \times 10^{-4} \mathrm{~cm}$. Finally, $\omega_{e e}, \omega_{e i}$, and $\omega_{e n}$ collision frequencies are $\omega_{e i}=1.32 \times 10^{9} \mathrm{Sec}^{-1}, \quad \omega_{e e}=9.34 \times$ $10^{8} \mathrm{Sec}^{-1}$, and $\omega_{e n}=2.399 \times 10^{-3} \mathrm{Sec}^{-1}$, correspondingly.

We explore the characteristics of GP in the presence of an applied electric field that varies in space and time and the impact of identical conditions as in [34]. We can study a comparison between different examples underneath identical conditions, one being the influence of a nonlinear applied magnetic field on the behavior of GP [34]. The second scenario investigates the impact of the NLAEF on GP electrons' behavior.

It is clear from the behavior of the distribution functions that the ascending particles and their turbulence after colliding with the plate are much more affected than the descending particles that have not yet collided with the plate, see Figs. (2A,2B). With the passage of time and the deviation of the system to equilibrium, we find that this disturbance in the behavior of the particles diminishes little by little; see the gradation and evolution of this behavior from $t=0.1,5,20,25.9$, see Figs. (2A,2B). The collision of the ascending turbulent particles with the unperturbed descending particles works to decay this behavior, seen gradually in Figs. (2A, 2B). Comparing the EVDF of turbulent electrons and their equilibrium ones shows that this turbulent behavior is more for the ascending particles than for the descending particles. That is very clear; see Figs. (3A,3B). The first component of the two-branched EVDF of descending particles deduces the tendency to equilibrium much faster than that of the ascending particles. That is directly affected by the movement of the plate and is also affected by the electromagnetic fields created, which of course, are as strong as possible near the plate forming it, see Figs. (3A,3B).

We can see from Figs. (1-A, B) that the NLAEF has a more substantial influence on GP electrons than the NLAMF [34], which swiftly pushes the GP out of its equilibrium case (EC) as $(t=2.5)$. The GP electrons achieve equilibrium over time, consistent with the laws of thermodynamics and the Le Chatelier criterion. When $(t=10)$, the GP electrons are a little out of equilibrium owing to NLAEF; see the last graph of Fig. (1-A), whereas it has achieved EC owing to NLAEF; check its last graph of Fig. (1) in Ref. [34].

We find that the NLAEF has a strong effect on GP. When contrasted to the action of the NLAMF, it causes it to vary and perturb aggressively. To keep the EC, we should use the impact of NLAMF rather than the impact of NLAEF in the GP controlling process.

Figures (2-A, B) show that the NLAEF has the impact of dramatically raising GP velocity in a short period. That is consistent with the perturbed EVDF's behavior. Away from RFP, the shear stress increases nonlinearly. The shear stress linearly behaves when it is next to the RFP; see Figs (3-A, B). The NLAMF, on the other hand, behaves nonlinearly adjacent to the surface [34].

The viscosity parameter $[29,46]$ performs linearly adjacent to the surface and nonlinearly far from it; see Figs (4-A, B). The NLAMF, on the other hand, behaves in a nonlinear fashion [34]. Figures (5-A, B) show how the NLAEF's nonlinear behavior influences GP electrons and how it is excessive (103 times) relative to the earlier study's comparable figure; see Fig. (5) in Ref. [34], since it was merely a generated magnetic field formed by RFP motion. It was found to be linked to NLAMF [34].

The behavior of the trustworthy induced magnetic field linked to the NLAEF compared to the NLAMF in [34] is depicted in Figures 6-A and B. The fluctuation and perturbation of GP can be explained in this 
way. When contrasted, we find that a high induced magnetic field is related to the NLAEF while a small electric field is connected to the NLAMF.

Boltzmann's H-theorem, thermodynamic principles, and Le Chatelier's concept agree that entropy increases with time. It is, however, slow-growing, which justifies the latency of GP electrons reaching an EC in this situation compared to the earlier study [34]; see Figs (7-A, B). In comparison to the NLAMF [34], we conclude that the pace of reaching an EC in the NLAEF instance is plodding. That supports the hypothesis that employing an NLAMF rather than an NLAEF is optimal for managing electron GP and transferring them to the EC.

Entropy generation is a positive function for all thermodynamics concepts, as seen in Figures 8-A and B. The IEM is shown in Figs. (9-A, B), (10-A, B), and (11, A, B) as a consequence of multiple factors; this compares in amplitude to the comparable figures in the prior research the following:

$$
\begin{aligned}
& d U_{S}(N E M F): d U_{S}(N E E F)=1: 10^{2}, \\
& d U_{P o l}(N E M F): d U_{P o l}(N E E F)=1: 4 \times 10^{3}, \\
& d U_{\text {dia }}(N E M F): d U_{\text {dia }}(N E E F)=1: 1.5 \times 10^{4} .
\end{aligned}
$$

As shown in [34], the IEM dominates in the NLAEF instance compared to the matching NLAMF cases. In the case of a research project:

$$
d U_{S}: d U_{P o l}: d U_{d i a}=1: 0.24 \times 10^{2}: 0.27 \times 10^{3} .
$$

Figures (14-A, B) show that with NLAEF, the gyrofrequency $\Omega_{c}$, decreases nonlinearly with space and time, whereas it decreases uniformly with space and time in the Ref. [34]. That is due to the strong electromagnetic fields occurring in this scenario. The gyroradius near the solid horizontal RFP shows a substantial perturbation in figure (14-A, B), whereas the NLAMF shows a smooth and consistent variation [34].

\section{CONCLUSIONS}

Plasmas are being applied in a wide range of applications. The ability to modify the GP's behavior via the NLAEF effect is key to these applications. For this, we contrasted the current work, which looks at GP behavior under the NLAEF effect, with the previous work, Ref. [34], which looked at GP behavior under the NLAMF effect. That was accomplished in the instance of a rigid plane surface moving in a GP. A rigorous analytical examination of the impact of timely and spatially variable applied electric fields on helium GP was carried out. In addition, the current displacement term in the Maxwell equations is regarded, which has hitherto been overlooked [17], [18], [20], [26], [28]. We developed a model to assess the GP's EVDF, velocity, and electromagnetic field. Obtained results correlate well with Boltzmann's Htheorem, thermodynamic principles, and Le Chatelier's rule. We conclude that:

1- The NLAEF has a strong influence on GP. In comparison to the impact of the NLAMF on it, it caused it to fluctuate and perturb aggressively; see [34]. To keep the EC, we should use the action of NLAMF rather than the effect of NLAEF in the GP process monitoring.

2- The NLAEF is related to a large induced magnetic field, whereas the NLAMF is connected to a mild induced electric field.

3- Compared to the NLAMF [34], the rate of reaching an EC in the NLAEF example is prolonged. That supports the idea that when controlling GP electrons to achieve an EC state, it is better to use an NLAMF rather than an NLAEF to get them there.

4- When compared with equivalent values in the situation of the NLAMF in [34], the IEM helps develop the NLAEF. In the case of a research project:

$$
d U_{S}: d U_{P o l}: d U_{\text {dia }}=1: 0.24 \times 10^{2}: 0.27 \times 10^{3} .
$$

5- All thermodynamic considerations are congruent with our model and all estimated variables.

Data Availability The data used to support the findings of this study are included in the article.

Conflict of Interest The author declares that there is no conflict of interest regarding the publication of this paper. 
Acknowledgments This study is supported by the Egyptian Academy of Scientific Research and Technology by the associated grant number (No.6508) under the ScienceUP Faculties of Science program.

\section{References}

[1] I. Adamovich et al. "The 2017 Plasma Roadmap: Low-temperature plasma science and technology. J. Phys. D: Appl. Phys. vol. 50, no.32, July. 2017.

[2] K.Weltmann et al., "The future for plasma science and technology," Plasma Processes and Polymers. vol. 16, Jan. 2019.

[3] A. Tejero et al., "The Lisbon Kinetics Boltzmann solver," Plasma Sources Sci. Technol., vol. 28, no. 4, pp. 1$21,2019$.

[4] Michele Campisi " Lectures on the Mechanical Foundations of Thermodynamics" Springer Nature Switzerland AG, (2021).

[5] Henry Clyde Foust, " Thermodynamics, Gas Dynamics, and Combustion," Springer Nature Switzerland AG, (2022).

[6] H. Arastoopour, D. Gidaspow, R. W. Lyczkowski " Transport Phenomena in Multiphase Systems." Springer Nature Switzerland AG, (2022).

[7] Abdel Wahid, T.Z., Elsaid, E.M., Morad, A.M. Exact solutions of plasma flow on a rigid oscillating plate under the effect of an external non-uniform electric field, Results in Physics, 19, 2020.

[8] A. M. Abourabia, and T. Z. Abdel Wahid. "Solution of the Krook Kinetic Equation Model and Non-Equilibrium Thermodynamics of a Rarefied Gas Affected by a Nonlinear Thermal Radiation Field," J. Non-Equilibrium Thermodynamics., vol. 36, no. 1, pp.75-98, May. 2011.

[9] A. M. Abourabia, and T. Z. Abdel Wahid "Kinetic and thermodynamic treatment for the Rayleigh flow problem of an inhomogeneous charged gas mixture," J. Non-Equilibrium Thermodynamics., Vol.37, no.1, pp.1-25, Mar. 2012.

[10] R Shahein, T. Z. Abdel Wahid "Advanced Problems in Plasma Physics" Saarbrücken, Germany: LAP LAMBERT Academic Publishing, ISBN-13: 978-620-4-72669-4, (December-2021).

[11] Abdel Wahid, T. Z., Exact analytical solution of the influence of an external centrifugal field and the heat transfer on a confined gas between two plates in the unsteady state, Advances in Mechanical Engineering, 12(11), 2020.

[12] Abdel Wahid, T. Z., Morad, A.M., On Analytical Solution of a Plasma Flow over a Moving Plate under the Effect of an Applied Magnetic Field, Advances in Mathematical Physics, 2020.

[13] Abdel Wahid, T. Z., On the irreversible thermodynamic of a gas influenced by a thermal radiation force generated from a heated rigid flat plate, Advances in Mechanical Engineering, 12(10), 2020.

[14] H. Arastoopour, D. Gidaspow, R. W. Lyczkowski " Transport Phenomena in Multiphase Systems." Springer Nature Switzerland AG, (2022).

[15] T. Z. Abdel Wahid, "Kinetic and thermodynamic treatment for the exact solution of the unsteady Rayleigh flow problem of a rarefied homogeneous charged gas," J. Non-Equilibrium Thermodynamics., vol.37, no.2, pp.119-141, Jun. 2012.

[16] T. Z. Abdel Wahid and SK. Elagan. "Kinetic Treatment for the Exact Solution of the Unsteady Rayleigh Flow Problem of a Rarefied Homogeneous Charged Gas Bounded by an Oscillating plate," Can. J. Phys., vol.90, pp.987998, 2012.

[17] T. Z. Abdel Wahid, "Travelling Waves Solution of the Unsteady Flow Problem of a Rarefied Nonhomogeneous Charged Gas Bounded by an Oscillating Plate," Math. Pro. in Eng., vol. 2013, 2013.

[18] A. M. Abourabia, and T. Z. Abdel Wahid. "Kinetic and thermodynamic treatments of a neutral binary gas mixture affected by a nonlinear thermal radiation field," Can. J. Phys., vol.90, pp.137-149, 2012.

[19] T. Z. Abdel Wahid. "Travelling Wave Solution of the Unsteady BGK Model for a Rarefied Gas Affected by a Thermal Radiation Field," Sohag J. Math., vol.2, no. 2, pp.75-87, 2015.

[20] T. Z. Abdel Wahid, "Travelling Waves Solution of the Unsteady Flow Problem of a Collisional Plasma Bounded by a Moving Plate," Fluid Mechanics., vol.4, no. 1, pp.27-37, Mar. 2018.

[21] L. Oliva, "Impact of the pre-equilibrium stage of ultra-relativistic heavy-ion collisions: isotropization and photon production," Eur. Phys. J. Plus., vol.134, 2019. 
[22] Halid Bikkin, Igor I. Lyapilin "Non-equilibrium Thermodynamics and Physical Kinetics” De Gruyter; 2nd edition (August 2, 2021).

[23] A. M. Abourabia, A. M. Morad. "Exact traveling wave solutions of the van der Waals normal form for fluidized granular matter," Phys. A., vol. 437, pp. 333-350, 2015.

[24] Hatim Machrafi "Extended Non-Equilibrium Thermodynamics: From Principles to Applications in Nanosystems" CRC Press; 1st edition (2019).

[25] T. Z. Abdel Wahid, "Exact solution of the unsteady Krook kinetic model and non-equilibrium thermodynamic study for a rarefied gas affected by a nonlinear thermal radiation field," Can. J. Phys., vol. 91, no.3, pp. 201-210, 2013.

[26] M. Baus, C. F. Tejero"Equilibrium Statistical Physics" Springer Nature Switzerland AG, (2021).

[27] G. M. Kremer, "An Introduction to the Boltzmann Equation and Transport Processes in Gases," SpringerVerlag Berlin Heidelberg, 2010.

[28] T. S. Chang, and C. M. Chang, "Rayleigh's problem in collisionless plasmas," Plasma Phys., vol.13, 1971.

[29] A. M. Abourabia, and R. E. Tolba, "On the irreversible thermodynamics of an electron gas in the vicinity of a suddenly moving rigid plate," Eur. Phys. J. Plus. Vol. 127, pp. 1-11, 2012.

[30] Adel M Morad, S. M. A. Maize, A. A. Nowaya, Y. S. Rammah "A New Derivation of Exact Solutions for Incompressible Magnetohydrodynamic Plasma Turbulence" Journal of Nanofluids, Vol. 10, No. 1, pp. 98-105(8), 2021.

[31] D. Pan, et al., "A unified gas kinetic scheme for transport and collision effects in plasma," Appl. Sci., vol. 8, 2018.

[32] J. Juno, et al., "Discontinuous Galerkin algorithms for fully kinetic plasmas," J. Comput. Phys., vol. 353, pp.110-147, 2018.

[33] H. Liu, et al., "Discrete unified gas kinetic scheme for a reformulated BGK-Vlasov-Poisson system in all electrostatic plasma regimes," Comp. Phys. Commun., vol.255, 2020.

[34] T. Z. Abdel Wahid, and A. M. Morad. "Unsteady plasma flow near an oscillating rigid plane plate under the influence of an unsteady nonlinear external magnetic field," IEEE Access., vol. 8, pp.76423-76432, May.2020.

[35] G. Lebon, D. Jou, and J. Casas-Vàzquez, "Understanding non-equilibrium thermodynamics: foundations, applications, frontiers," Springer-Verlag, Berlin, Heidelberg, Germany, 2008.

[36] T. Z. A. Wahid, On Irreversible Thermodynamic for a New Collision Frequency Model of Boltzmann Equation for a Gas Mixture Influenced by a Centrifugal Force, Journal of Statistics Applications \& Probability, Vol. 10, No. 3 (Nov. 2021), PP:897-903.

[37] J. D. Huba, "NRL plasma formulary," Naval Research Laboratory, Washington, DC, 2019.

[38] S. I. Braginskii, "Transport processes in a plasma. Reviews of Plasma Physics, vol. 1, Authorized translation from Russian by Herbert Lashinsky, University of Maryland, USA. Edited by M. A. Leontovich. Published by Consultants Bureau, New York, pp.205, 1965.

[39] L. Lees, "Kinetic theory description of rarefied gas flow," J. Soc. Ind. Appl. Math., vol.13, 1965.

[40] A. M. Abourabia, T. S. El-Danaf, and A. M. Morad. "Exact solutions of the hierarchical Korteweg-de Vries equation of microstructured granular materials," Chaos, Solitons \& Fractals vol. 41, pp. 716-726, 2009.

[41] J. Gratton, S. M. Mahajan, and F. Minotti. "Non-Newtonian Gravity Creeping Flow," International Centre for Theoretical Physics, Trieste (Italy), pp.1-17 (1988).

[42] G. Nugroho, A. M. S. Ali, and Z. A. Abdul Karim. "Towards a new simple analytical formulation of NavierStokes Equations," World Acd. Sci. Eng. and Tec., vol. 51, 2009.

[43] Wahid, T.Z.A., El-Malky, F.M. Thermodynamic and kinetic investigation of the influence of external centrifugal field and the heat transfer on a confined neutral gas. SN Appl. Sci. 2, 791 (2020). https://doi.org/10.1007/s42452-020-2583-9

[44] P. Van der Linde, "Periodica Polytechnica Ser," Chem. Eng. 12, 97 (1998).

[45] M. Boulos, P. Fauchais, E. Pfender, Handbook of Thermal Plasmas. Springer International Publishing AG, part of Springer Nature 2018.

[46] A. M. Abourabia, and T. Z. Abdel Wahid. "The unsteady Boltzmann kinetic equation and non-equilibrium thermodynamics of an electron gas for the Rayleigh flow problem" Canadian Journal of Physics 88 (7), 501-511, 2010 . 

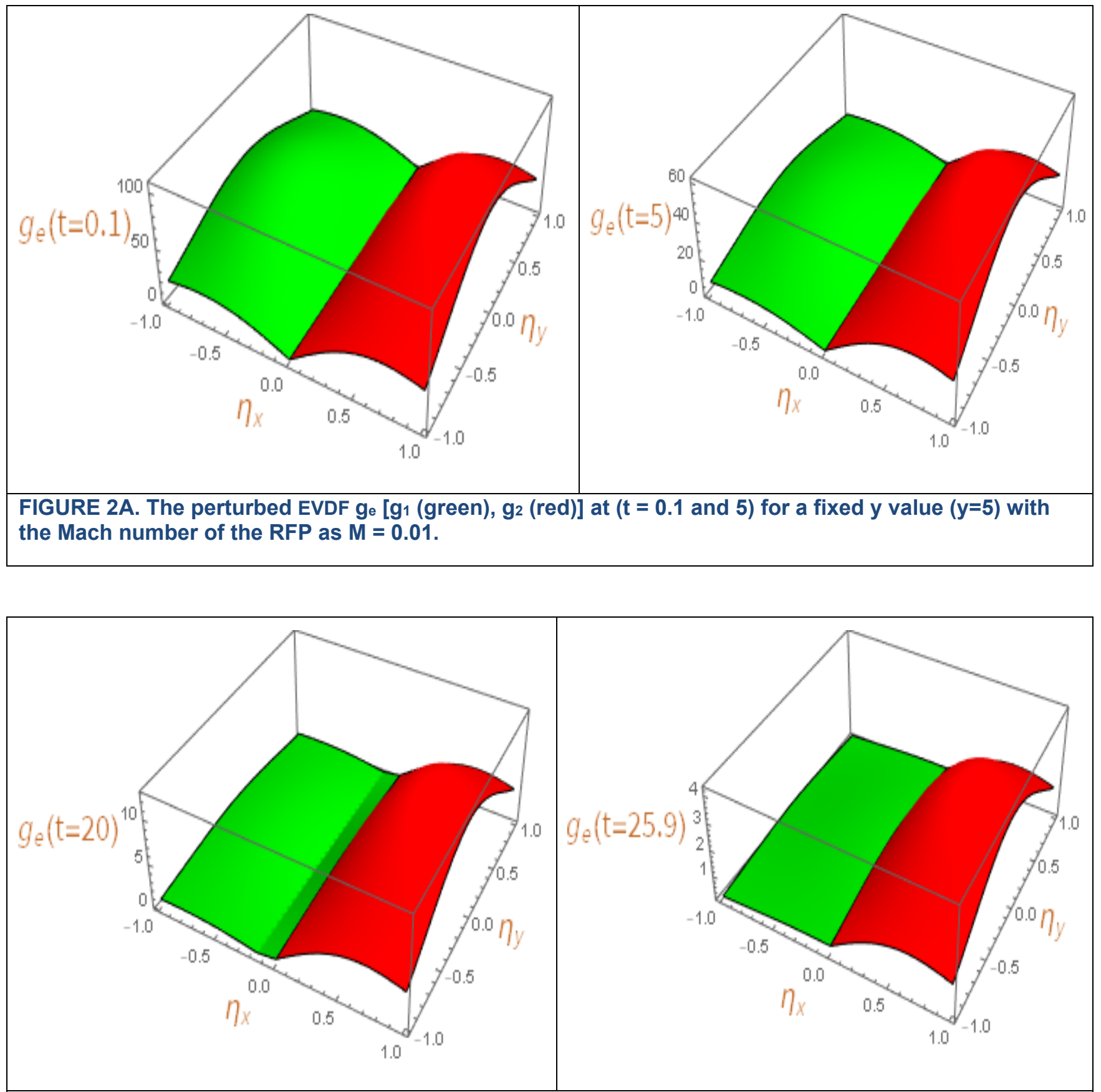

FIGURE 2B. The perturbed EVDF $g_{\mathrm{e}}$ [g $\mathrm{g}_{1}$ (green), $\mathrm{g}_{2}$ (red)] at $(\mathrm{t}=20$ and 25.9) for a fixed y value $(\mathrm{y}=5)$ with the Mach number of the RFP as $M=0.01$. 


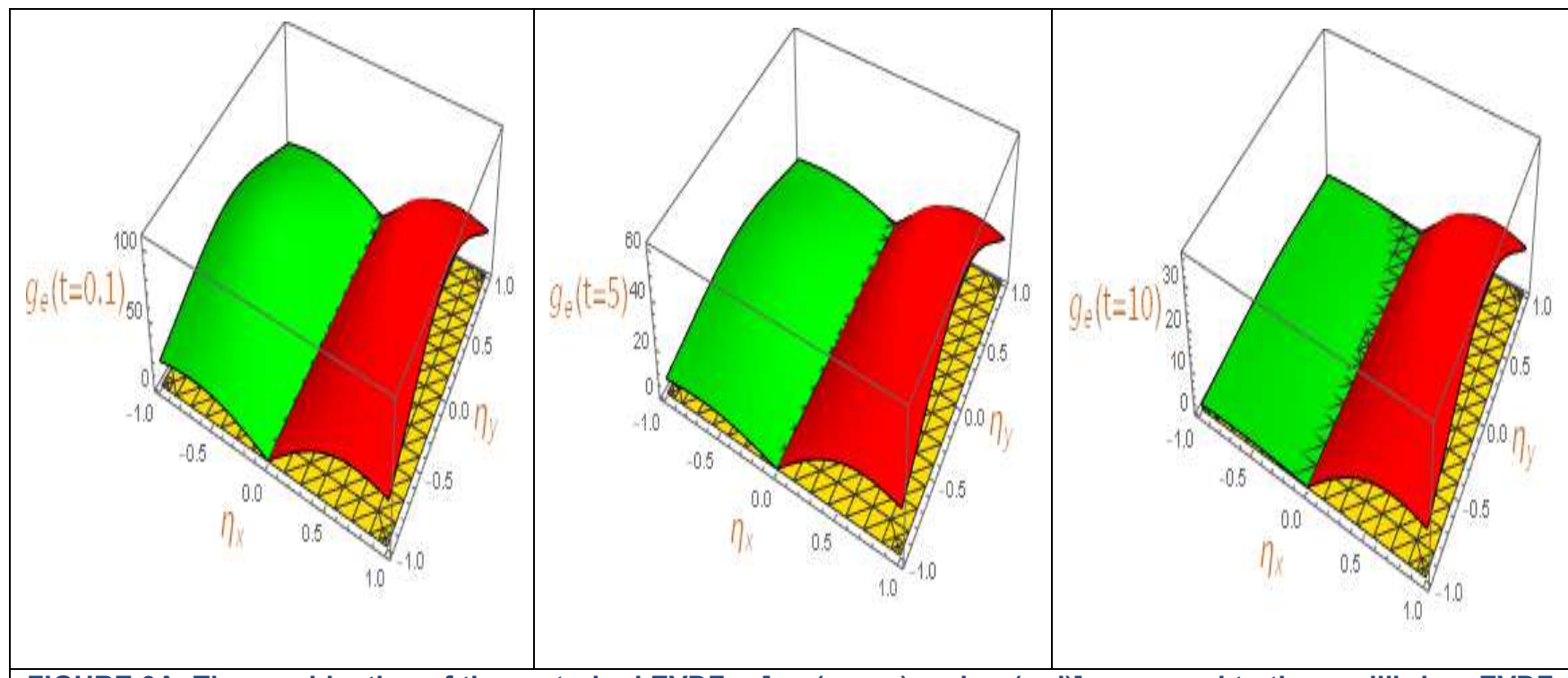

FIGURE 3A. The combination of the perturbed EVDF $\mathrm{g}_{\mathrm{e}}\left[\mathrm{g}_{1}\right.$ (green) and $\mathrm{g}_{2}$ (red)] compared to the equilibrium EVDF go (grid) at $(t=0.1,5$, and 10$)$ with the Mach number of the RFP $M=0.01$ at $y=5$.

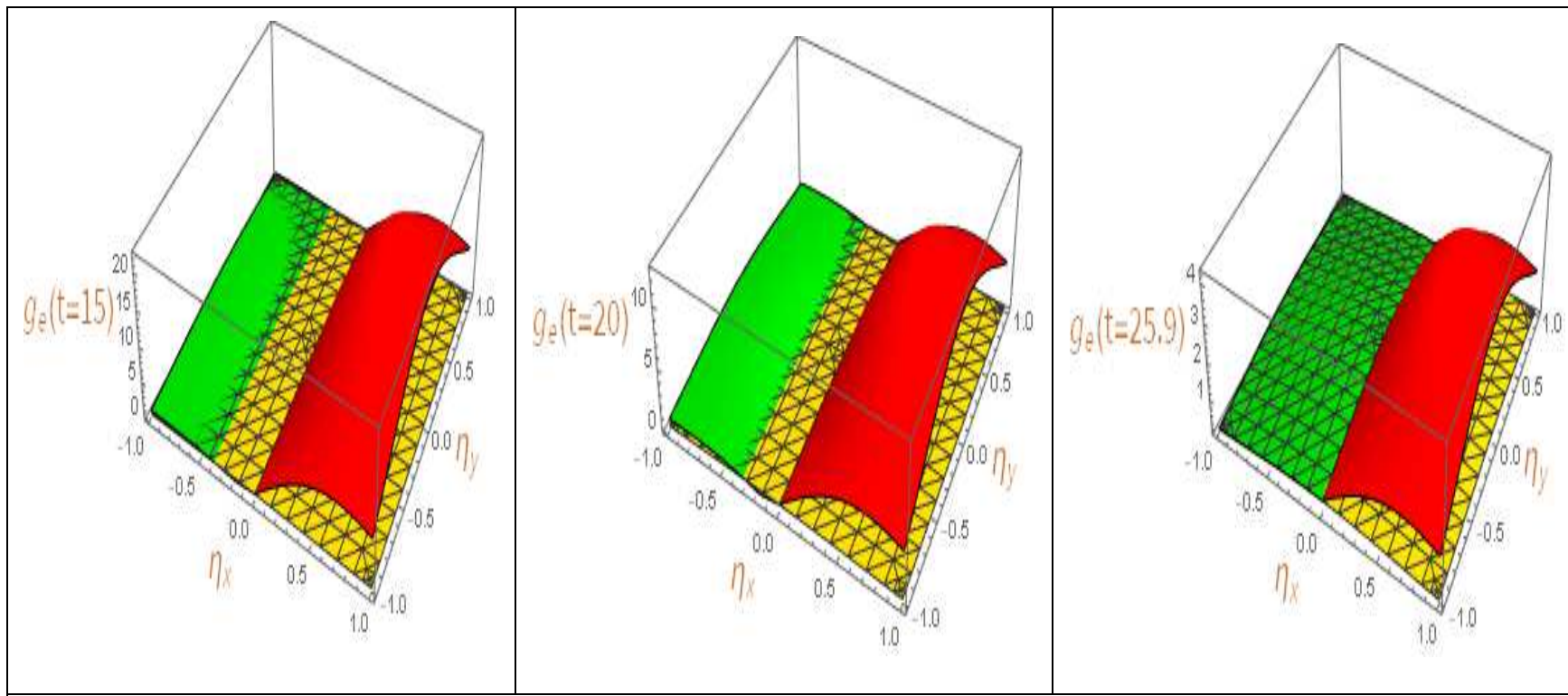

FIGURE 3B. The combination of the perturbed EVDF $g_{e}\left[g_{1}\right.$ (green) and $g_{2}$ (red) ] compared to the equilibrium EVDF g。 (grid) at $(t=15,20$, and 25.9) with the Mach number of the RFP $M=0.01$ at $y=5$. 


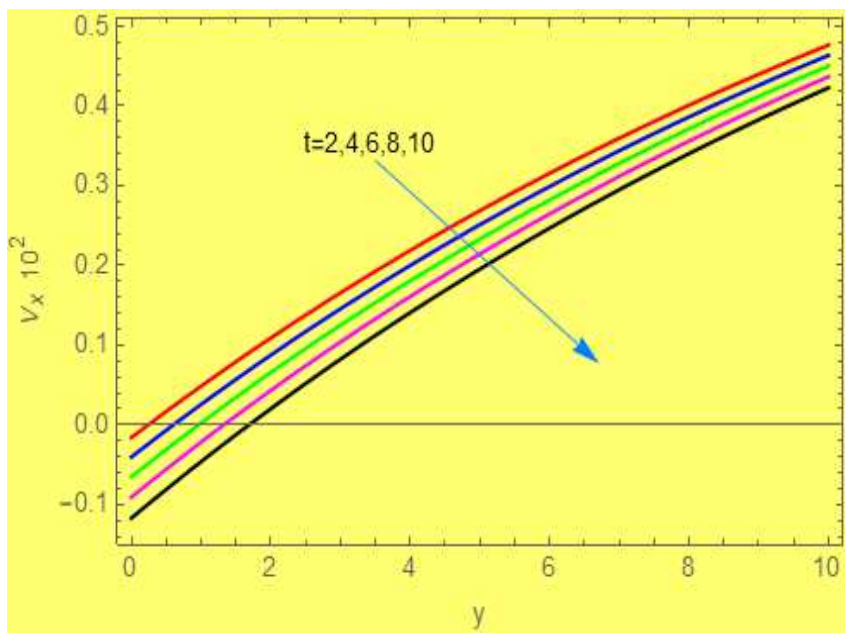

FIGURE 4A. The Velocity profiles Vx with $y$ for various values of $t$.

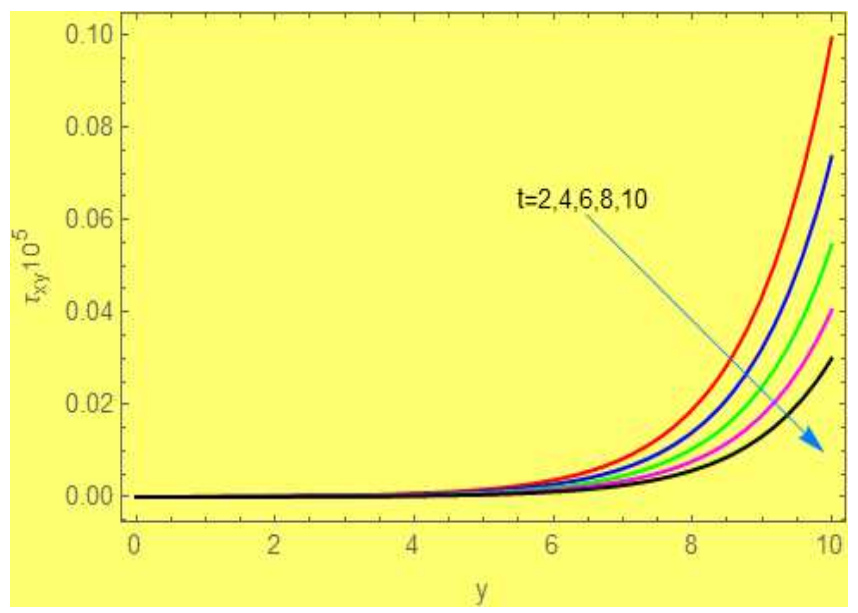

FIGURE 5A. The shear stress profiles $\tau_{x y}$ with $\mathrm{y}$ for various values of $\mathrm{t}$.

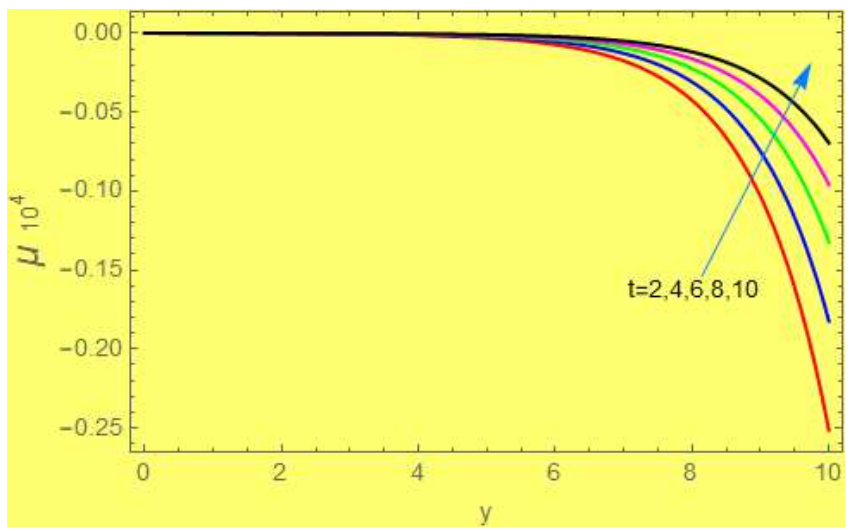

FIGURE 6A. The viscosity coefficient profiles $\mu$ with y for various values of $t$.

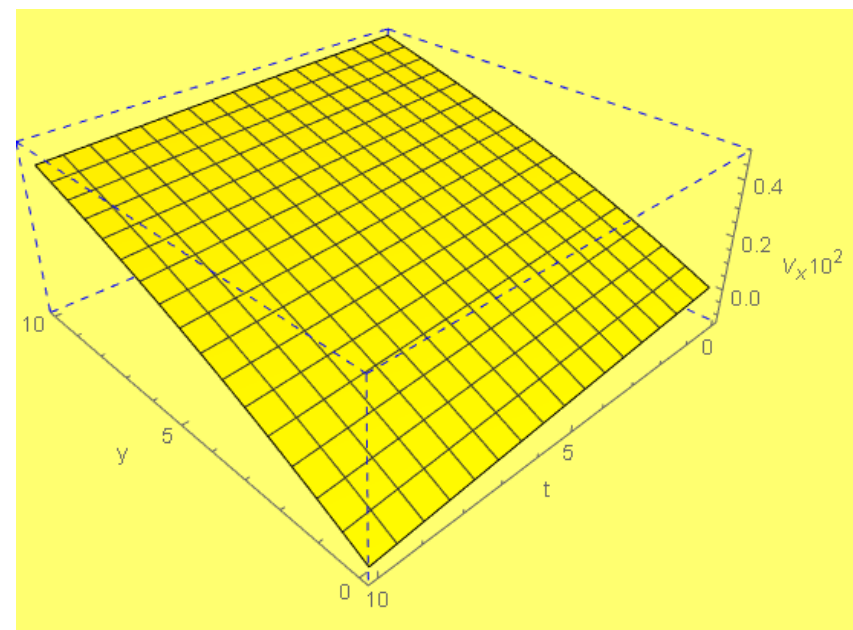

FIGURE 4B. The velocity profile Vx vs. $y$ and $t$.

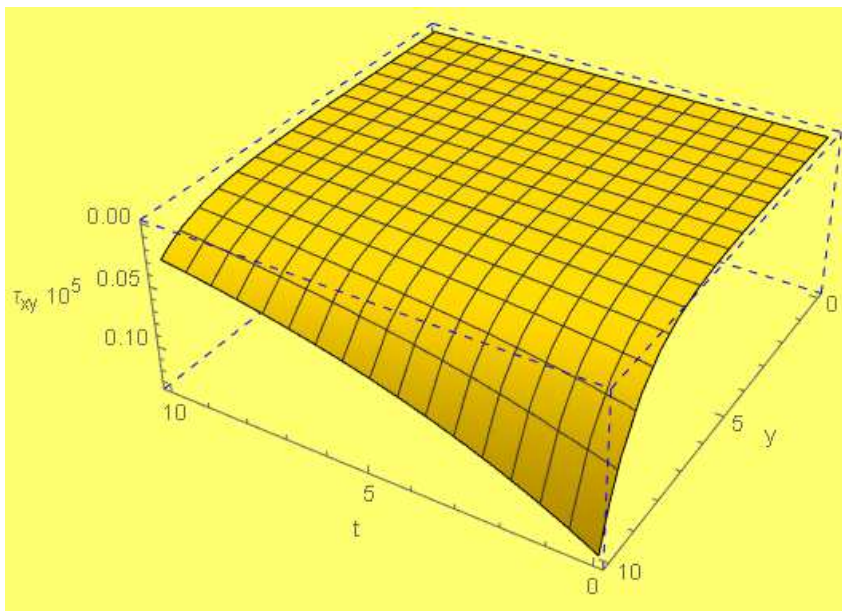

FIGURE 5B. The shear stress $\tau_{x y}$ vs. $y$ and t.

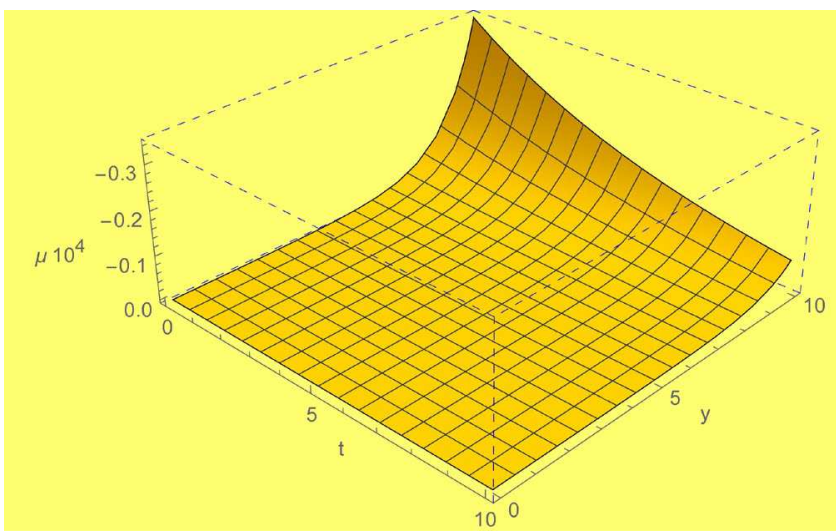

FIGURE 6B. The viscosity coefficient $\mu$ vs. y and t. 


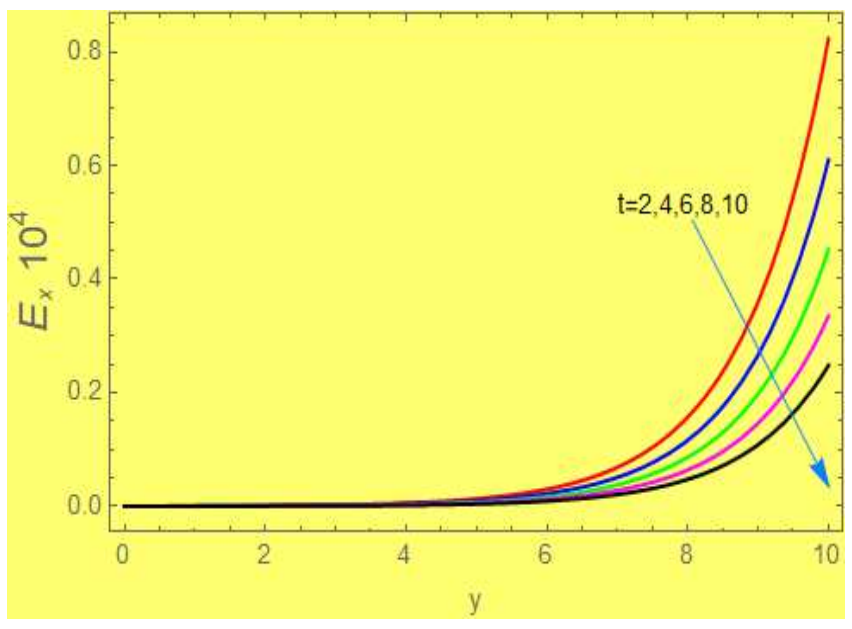

FIGURE 7A. The applied electric field profiles Ex with y for various $t$.

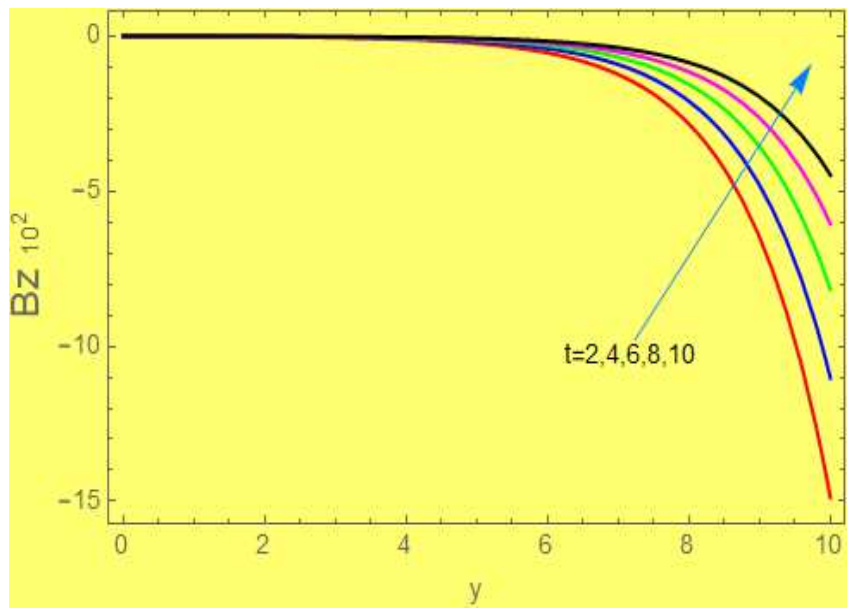

FIGURE 8A. The induced magnetic field profiles $\mathrm{Bz}$ with $\mathrm{y}$ for various values of $t$.

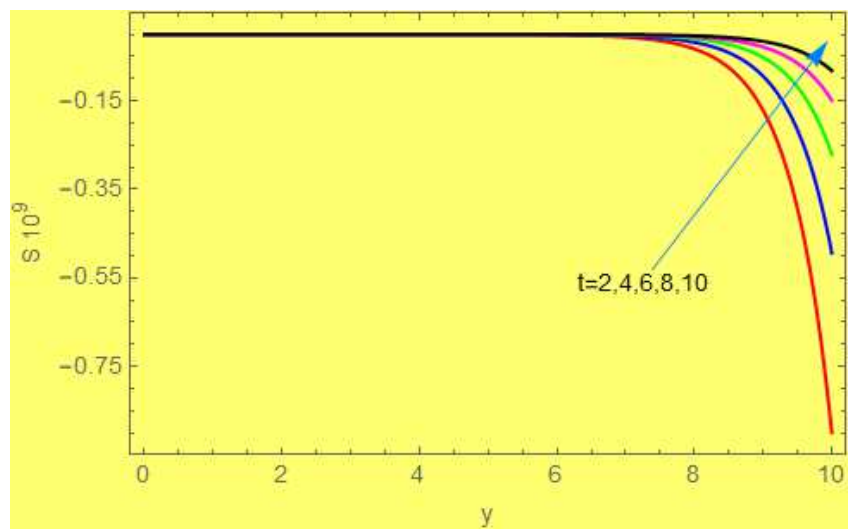

FIGURE 9A. The entropy profiles $S$ with $y$ for various values of $t$.

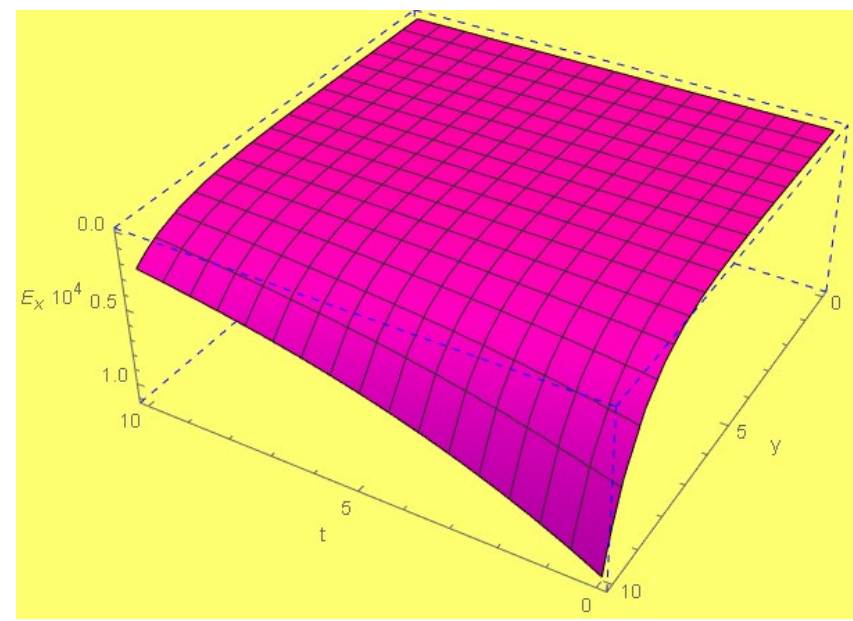

FIGURE 7B. The applied electric field Ex vs. $y$ and $t$.

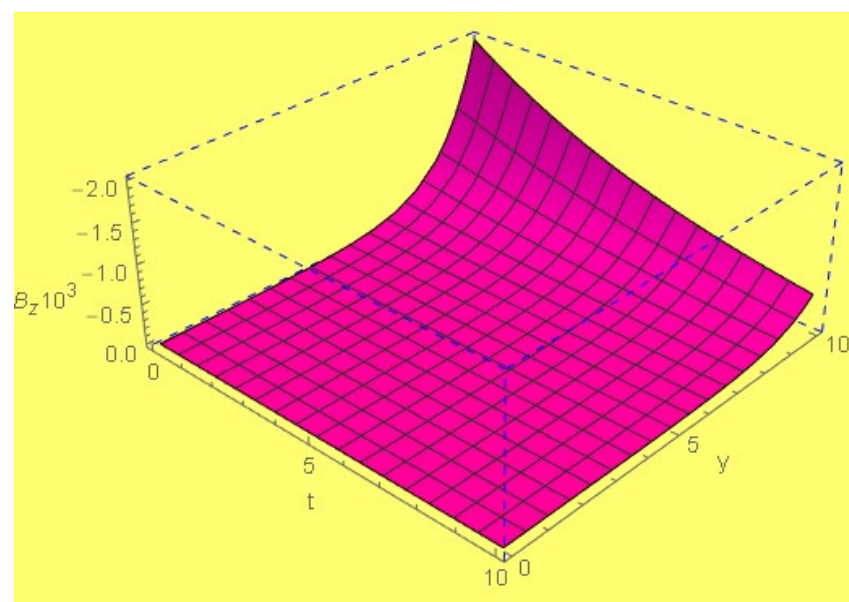

FIGURE 8B. The induced magnetic field $B z$ vs. $y$ and $t$.

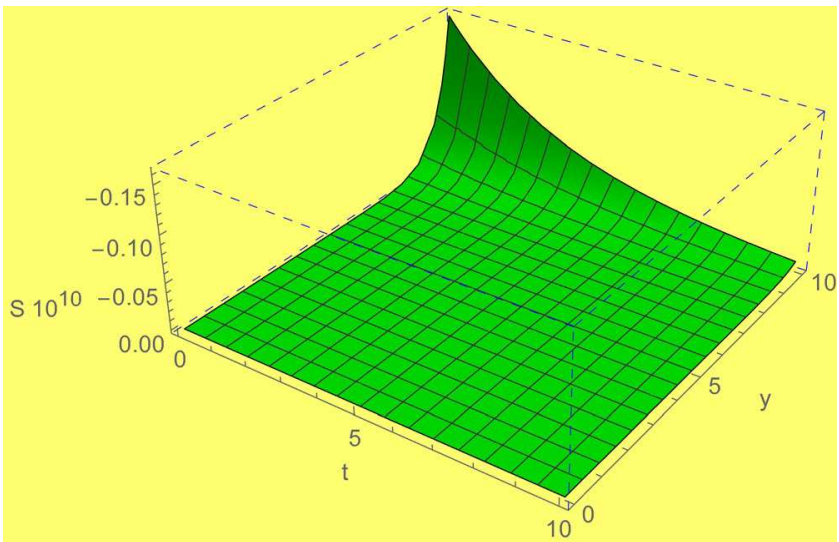

FIGURE 9B. The entropy $S$ vs. $y$ and $t$. 


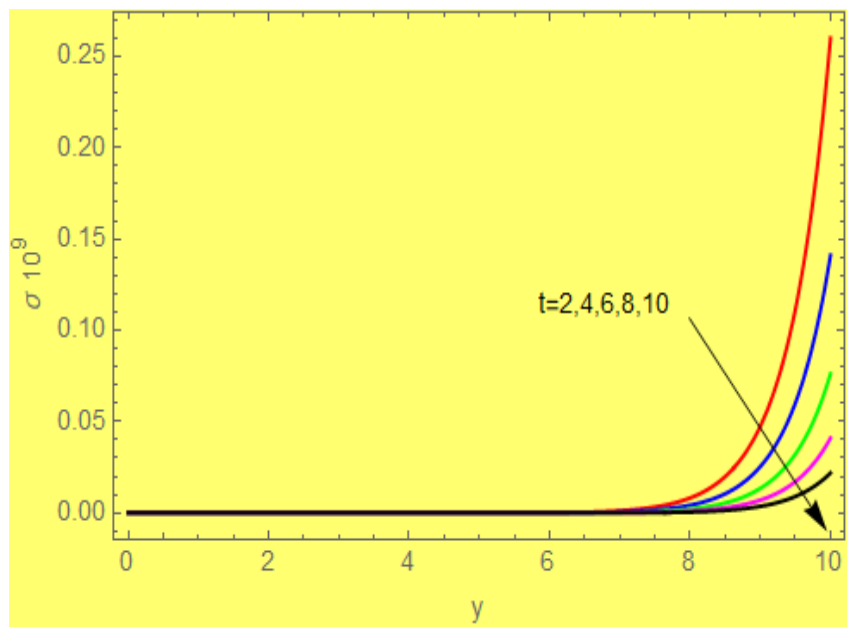

FIGURE 10A. The entropy production profiles $\sigma$ with y for various values of $t$.

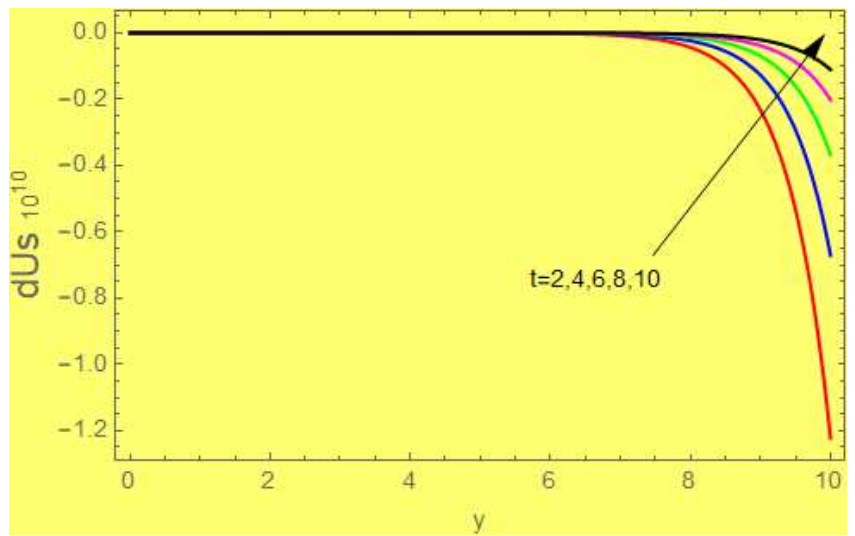

FIGURE 11A. The IEM dU $\mathrm{d}_{\mathrm{s}}$ profile with $\mathrm{y}$ for various values of $\mathrm{t}$.

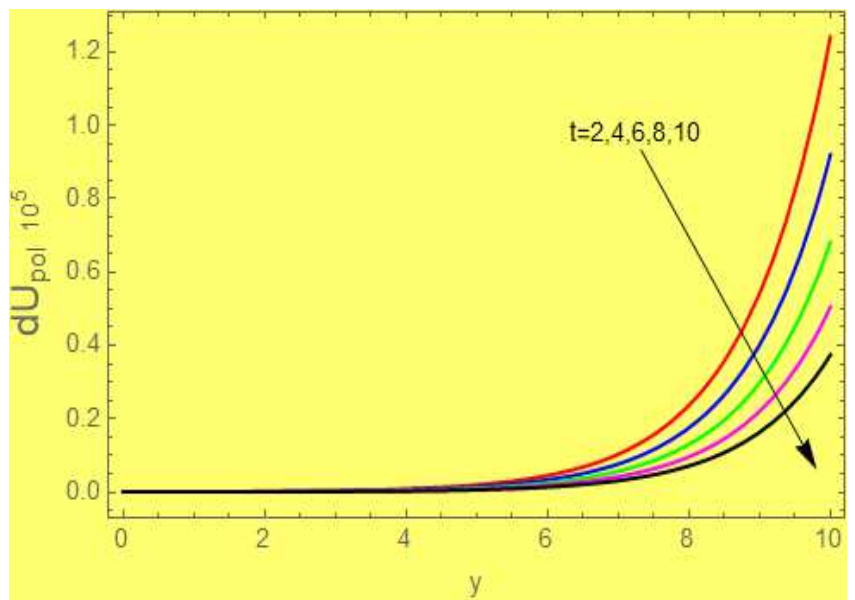

FIGURE 12A. The IEM dU pol profile with $y$ for various values of $t$.

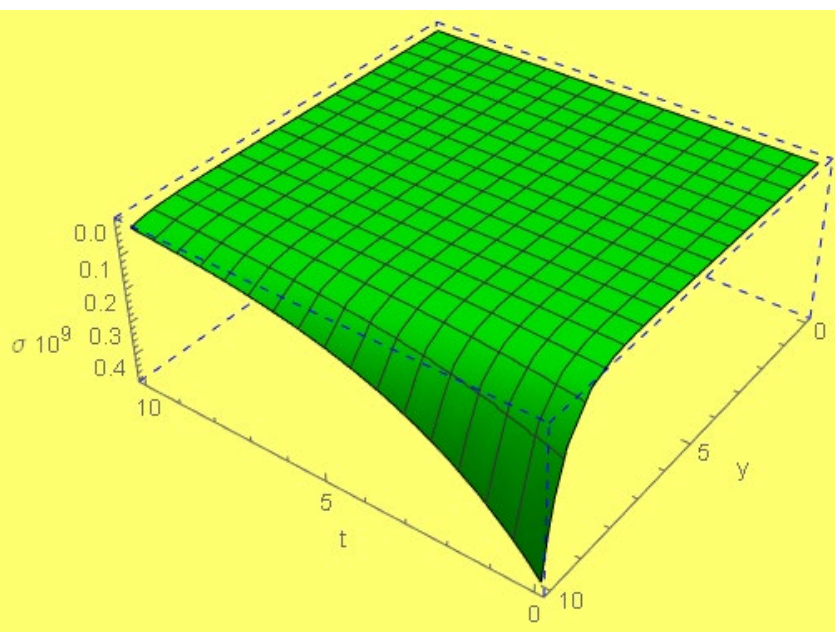

FIGURE 10B. The entropy production $\sigma$ vs. $y$ and $t$.

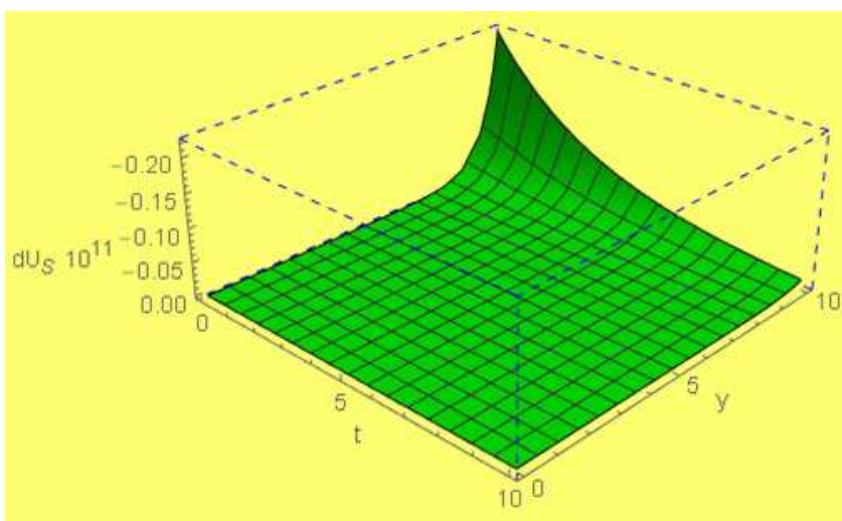

FIGURE 11B. The IEM dU s vs. y and t.

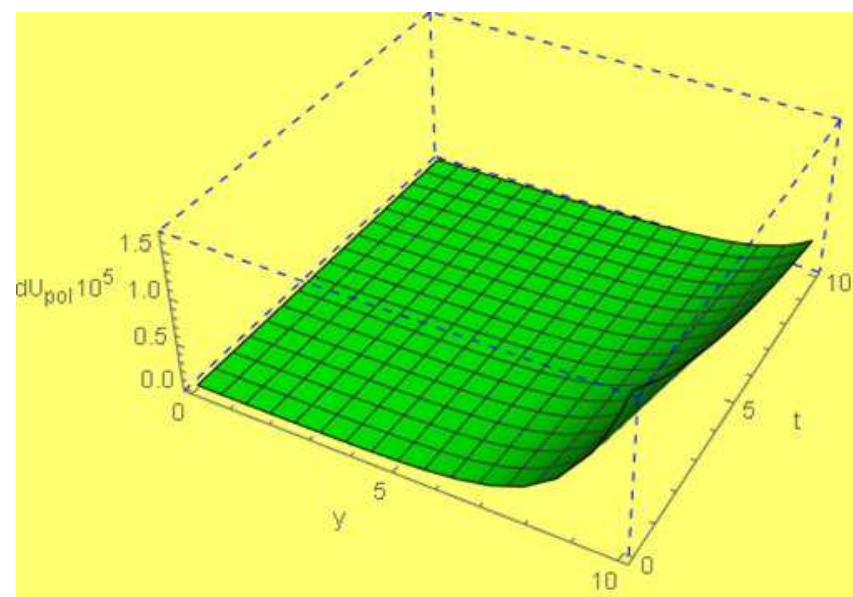

FIGURE 12B. The IEM $\mathrm{dU}_{\mathrm{pol}}$ vs. $y$ and $t$. 


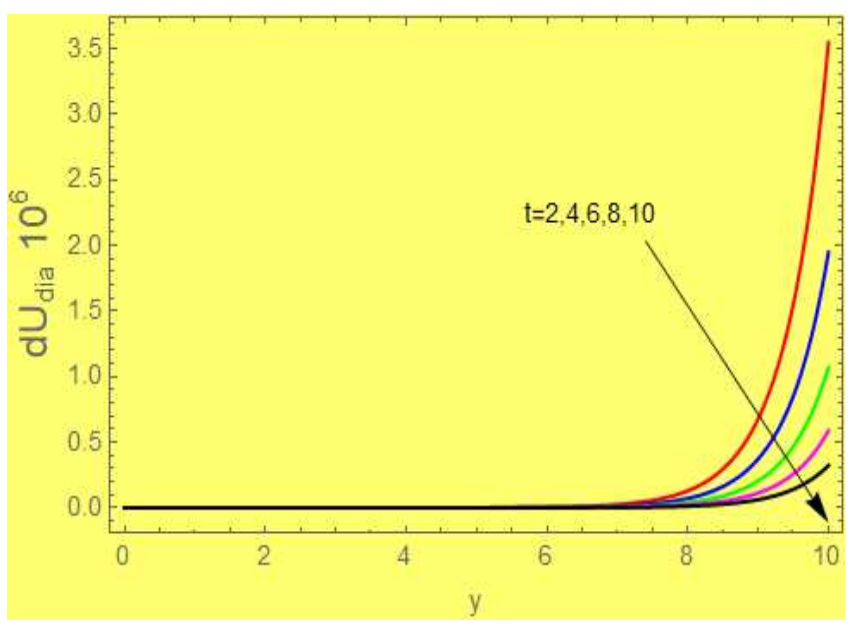

FIGURE 13A. The IEM dU dia profile with $y$ for various values of $t$.

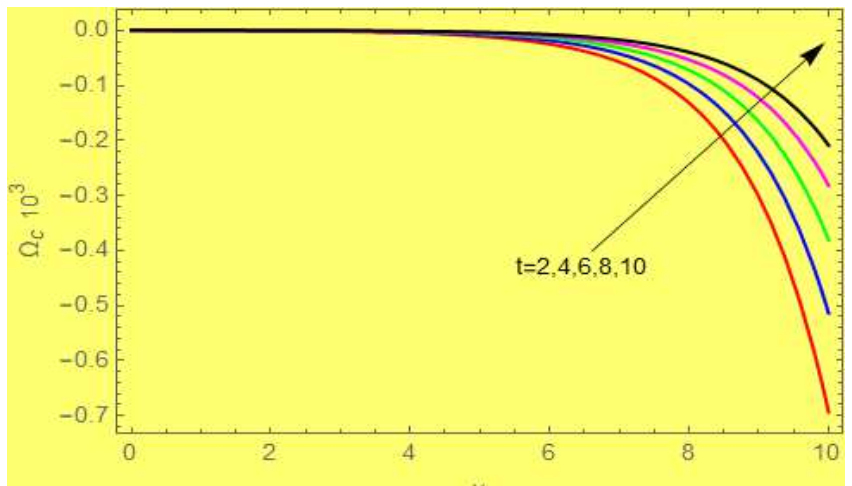

FIGURE 14A. The gyro-frequency $\Omega_{c}$ profile with $y$ for various values of

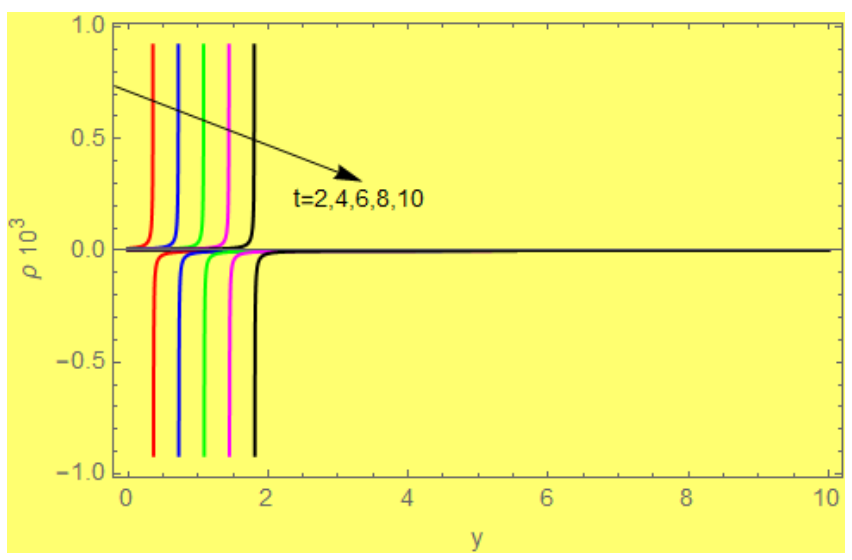

FIGURE 15A. The gyro-radius $\rho$ profile with y for various values of $t$.

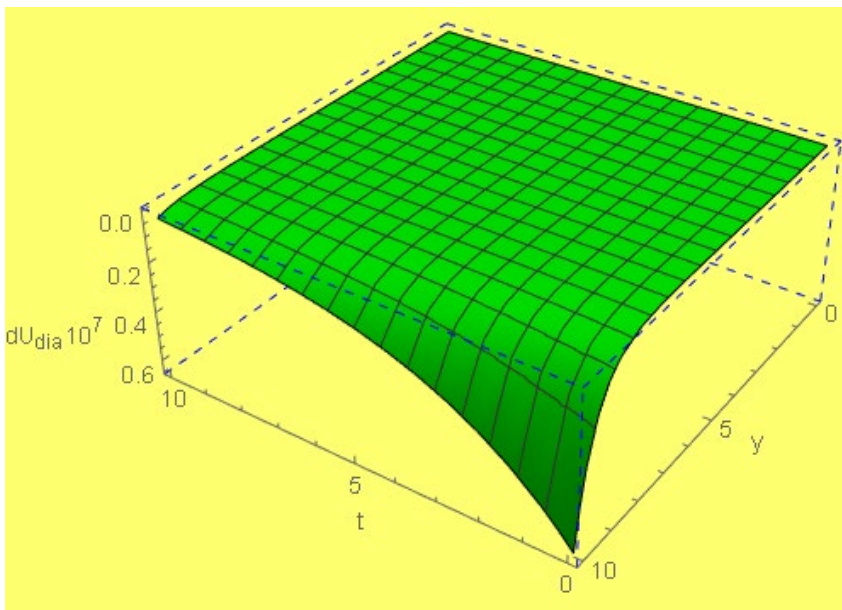

FIGURE 13B. The IEM dUdia vs. $y$ and $t$.

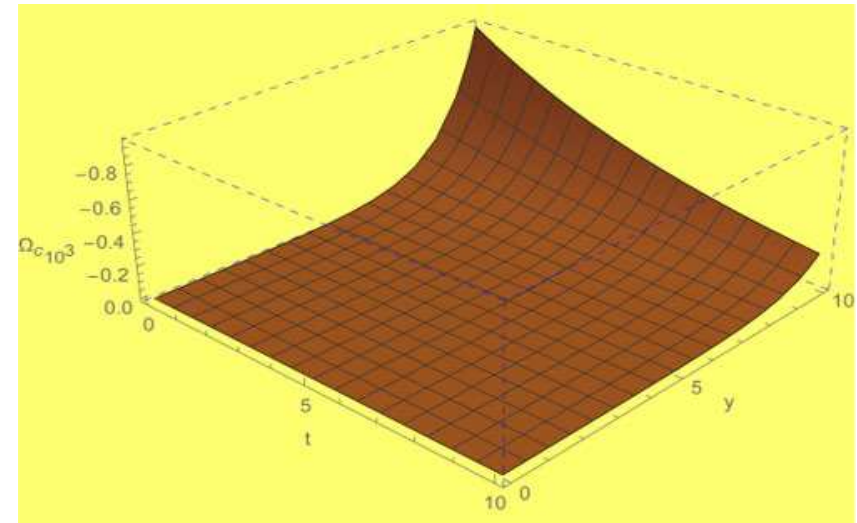

FIGURE 14B. The gyro-frequency $\Omega_{c}$ vs. y and t.

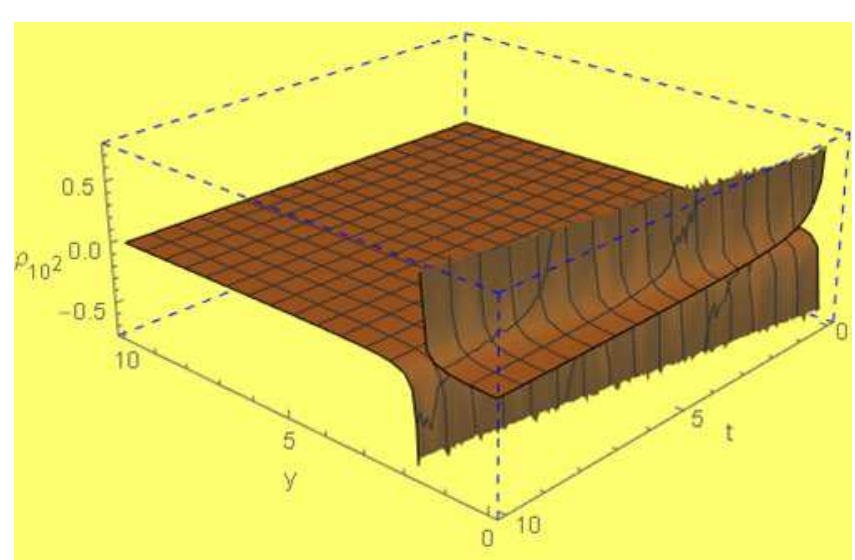

FIGURE 15B. The gyro-radius $\rho$ vs. $y$ and $t$. 


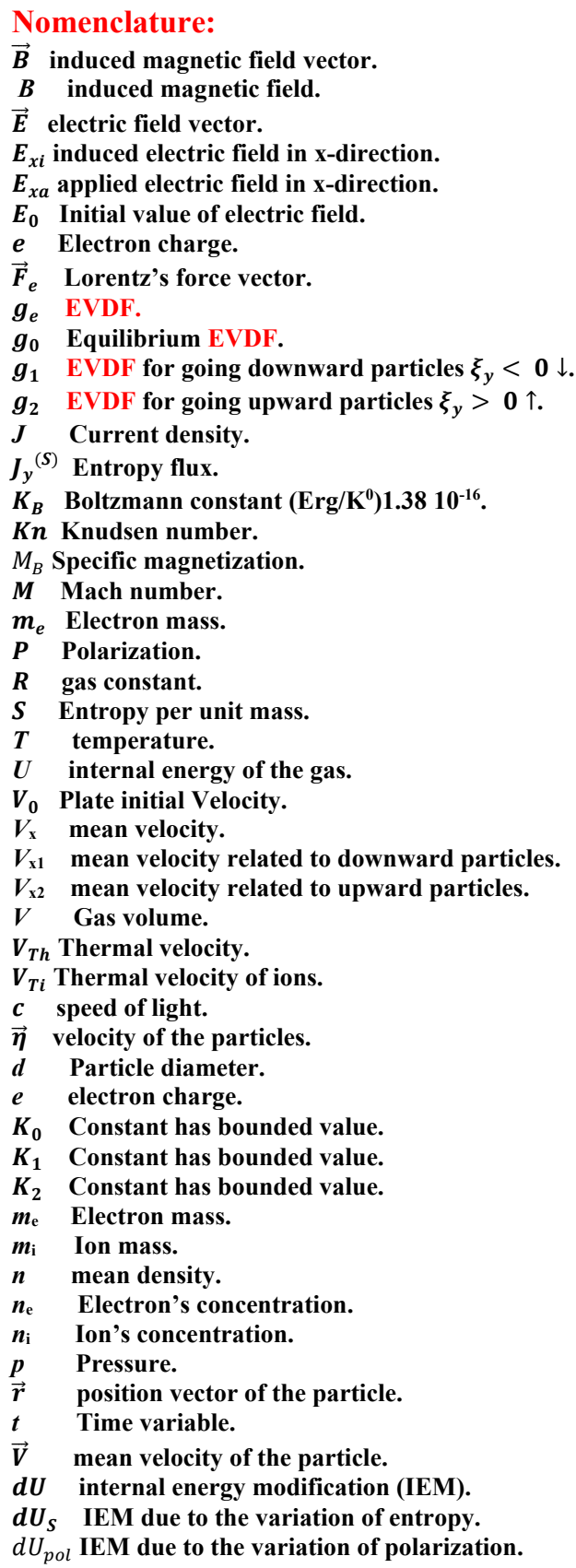

$d U_{P a r}$ IEM due to variation of magnetization.

$d U_{\text {dia }}$ IEM due to variation of magnetic field.

$y$ Displacement variable.

$Z \quad$ Ionization degree.

\section{Superscripts:}

* Dimensionless variable.

\section{Subscripts:}

$e$ for electrons.

$i$ for ions.

$n$ for neutral atoms.

$x \quad$ in $\mathrm{x}$-direction.

$y \quad$ in $\mathbf{y}$-direction.

$z \quad$ in z-direction.

$\alpha \quad=\mathrm{e}$ for electrons or $=\mathbf{i}$ for ions

\section{Greek letters:}

$\vartheta_{j}(\vec{\xi})$ Functions of velocity and $j: 1$ to $n$, here $n$ is the number of the moments.

$\gamma \quad$ Travelling wave parameter.

$\tau$ relaxation time.

$\tau_{e e}$ Electron-electron relaxation time.

$\tau_{e i} \quad$ Electron-ion relaxation time.

$\tau_{e n} \quad$ Electron-neutral relaxation time.

$\tau_{x y} \quad$ Shear stress.

$\mu \quad$ Viscosity coefficient.

$\lambda$ mean free path.

$\lambda_{D} \quad$ Debye length.

$\omega$ Frequency.

$\Omega_{e 0} \quad$ Non-dimensional parameter.

$\omega_{e e}$ e-e Collision frequency.

$\omega_{e i} \quad$ e-i Collision frequency.

$\omega_{e n}$ e-n Collision frequency.

mei Electron-ion mass ratio.

$\lambda \quad$ Mean free path.

$\lambda_{D} \quad$ Debye radius.

$\sigma \quad$ Entropy production or entropy generation.

$\log [\Lambda]$ Coulomb logarithm.

Abbreviations

BGK Bhatnagar-Gross-Krook.

BE Boltzmann equation.

EC Equilibrium Case.

EVDF Electron Velocity distribution function.

GP Gaseous plasma.

IEM Internal Energy Modification.

INTD Irreversible Non-Equilibrium Thermodynamics.

MEMS Micro-Electro-Mechanical Systems.

MM Moment method

NLAEF Nonlinear Applied Electric Field.

NLAMF Nonlinear Applied Magnetic Field.

NEMS Nano-Electro-Mechanical Systems. 